\title{
SUR LE VOLUME DES ZÉROS DES FONCTIONS HOLOMORPHES ET BORNÉES DANS LA BOULE DE $\mathbf{C}^{n}$
}

\author{
ERIC AMAR
}

\begin{abstract}
We give an example of a zero set of a holomorphic bounded function in the unit ball of $\mathbf{C}^{n}, n \geqslant 2$, with infinite area. This generalizes a previous work of $\mathrm{W}$. Rudin done in the case $n=2$.
\end{abstract}

Introduction. B. Berndtsson proves in [2] that a holomorphic variety in the unit ball of $\mathbf{C}^{2}$ of finite area is the zero set of a bounded holomorphic function in $\mathbf{B}_{2}$.

W. Rudin [5] gives an example of a zero set of a holomorphic bounded function in $\mathbf{B}_{2}$ with infinite area. In this work we generalize the previous work of W. Rudin in the unit ball $\mathbf{B}_{n}$ of $\mathbf{C}^{n}$; we use a result of $\mathbf{N}$. Varopoulos [7] concerning the zero set of holomorphic functions in the Hardy classes $H^{P}\left(\mathbf{B}_{n}\right)$.

We construct a holomorphic variety $X$ in $\mathbf{B}_{n}$ such that

THEOREM. i. $X$ is of infinite area.

ii. $X$ is the zero set of a bounded holomorphic function in $\mathbf{B}_{n}$.

iii. If we throw out a component of $X$, then the remaining holomorphic variety is not the zero set of a bounded holomorphic function in $\mathbf{B}_{n}$.

In fact $X$ is the zero set of a holomorphic function $e^{k}\left(\overline{\mathbf{B}}_{n}\right)$ for all $k \in \mathbf{N}$, but I do not know if $X$ is the zero set of a holomorphic function in $e^{\infty}\left(\overline{\mathbf{B}}_{n}\right)$.

1. Rappelons quelques définitions:

Pour $p>0$, on note $A^{p}\left(\mathbf{B}_{n}\right)$ les classes de Bergman de la boule

$$
A^{p}\left(\mathbf{B}_{n}\right)=\left\{f \text { holomorphe dans } \mathbf{B}_{n} / \int_{\mathbf{B}_{n}}|f(z)|^{p} d v(z)=\|f\|_{p}^{p}<+\infty\right\}
$$

où $d v$ est le volume euclidien de $\mathbf{B}_{n}$; et les classes de Hardy

$$
\begin{aligned}
& H^{\infty}\left(\mathbf{B}_{n}\right)=\left\{f \text { holomorphe et bornée dans } \mathbf{B}_{n} ;\|f\|_{\infty}=\sup _{z \in \mathbf{B}_{n}}|t(z)|\right\} \\
& H^{p}\left(\mathbf{B}_{n}\right)=\left\{f \text { holomorphe dans } \mathbf{B}_{n} / \sup _{r<1} \int_{|z|=1}|f(r z)|^{p} d \sigma(z)=\|f\|_{p}^{p}<+\infty\right\}
\end{aligned}
$$

où $d \sigma$ est la mesure de Lebesgue sur $S=\partial \mathbf{B}_{n}$; enfin la classe de Nevanlinna

$$
N\left(\mathbf{B}_{n}\right)=\left\{f \text { holomorphe dans } \mathbf{B}_{n} / \sup _{r<1} \int_{|z|=1} \log ^{+}|f(r z)| d \sigma(z)<+\infty\right\} .
$$

Received by the editors March 29, 1981 and, in revised form. July 28, 1981.

1980 Mathematics Subject Classification. Primary 32A35: Secondary 62B40.

(C) 1982 American Mathematical Society 0002-9939/81/0000-1113/\$02.00 
Si $X$ est un ensemble analytique dans $\mathbf{B}_{n}$, on note $[X]$ la mesure d'intégration sur $X[4]$.

On va montrer que le théorème se déduit de la proposition suivante, proposition que l'on démontrera au paragraphe 2 . La preuve que la proposition entraine le théorème est essentiellement due à W. Rudin.

Proposition. Il existe un ensemble analytique $X$ dans $\mathbf{B}_{n}$ qui est le zéro d'une fonction d'une classe de Bergman mais qui n'est pas le zéro d'une fonction de la classe de Nevanlinna.

Admettons donc cette proposition et prouvons le théorème.

Soit $X$ l'ensemble analytique donné par la proposition; puisque $X$ n'est pas un diviseur de la classe $N\left(\mathbf{B}_{n}\right)$ on a

$$
\int_{\mathbf{B}_{n}}\left(1-|z|^{2}\right)[X]=+\infty .
$$

Plongeons $X$ dans $\mathbf{B}_{n+1}$ ainsi

$$
\tilde{X}=\left\{(z, w) \in \mathbf{B}_{n+1} / z \in X\right\}
$$

et soit

$$
D(z)=\left\{w \in \mathbf{C}, 1-|z|^{2}>|w|^{2}\right\}, \quad z \in \mathbf{B}_{n} .
$$

Par Fubini il vient alors

$$
\int_{\mathbf{B}_{n+1}}[\tilde{X}]=\int_{\mathbf{B}_{n}}[X]\left\{\int_{D(z)} d \lambda(w)\right\}=\int_{\mathbf{B}_{n}}\left(1-|z|^{2}\right)[X]=+\infty
$$

où $d \lambda(w)$ est la mesure de Lebesgue de $\mathbf{C}=\mathbf{R}^{2}$.

D'autre part, pour $p>0$, si $f \in A^{p}\left(\mathbf{B}_{n}\right)$ on a [5, Théorème 7.2.5]

$$
\exists C>0, \exists k \in \mathbf{N}, \text { t.q. }|f(z)| \leqslant \frac{C}{\left(1-|z|^{2}\right)^{k}}, \quad z \in \mathbf{B}_{n} .
$$

Pour conclure le théorème, on fait alors comme Walter Rudin [5]; si $f$ est la fonction de $A^{p}\left(\mathbf{B}_{n}\right)$ qui s'annule sur $X$ on pose

$$
F_{l}(z, w)=w^{2 k+l+1} f(z), \quad(z, w) \in \mathbf{B}_{n+1}, l \in \mathbf{N} .
$$

On a alors que

(1) $F_{l} \in H^{\infty}\left(\mathbf{B}_{n+1}\right) \cap \bigcup^{\prime}\left(\overline{\mathbf{B}}_{n+1}\right)$

car dans $\mathbf{B}_{n+1}$ on $\mathbf{a}|w|^{2} \leqslant\left(1-|z|^{2}\right)$,

(2) $Y=F_{l}^{-1}(0)=\tilde{X} \cup\{w=0\}$ donc vol $Y \geqslant \operatorname{vol} \tilde{X}=+\infty$,

(3) $\tilde{X}$ n'est pas le zéro d'une fonction de $H^{\infty}\left(\mathbf{B}_{n+1}\right)$; sinon soit $\tilde{h}(z, w)$ cette fonction, alors la fonction

$$
z \in \mathbf{B}_{n}, \quad h(z)=\tilde{h}(z, 0)
$$

serait encore dans $H^{\infty}\left(\mathbf{B}_{n}\right)$ et s'annulerait sur $X$, ce qui n'est pas possible, $X$ ne satisfaisant pas à la condition de Blaschke par (1.1).

L'ensemble $Y$ vérifie donc les conclusions du théorème. 


\section{Preuve de la proposition.}

Notations. (*) $a \lesssim b$ si il existe une constante $C$ dépendant au plus de la dimension telle que $a \leqslant C b$,

$(* *) a \simeq b$ si $a \lesssim b$ et $b \lesssim a$.

Rappels. Soit $S=\partial \mathbf{B}_{n}$ le bord de la boule; on définit sur $S$ la pseudo-distance de Koranyi [3; 1, Chapter IV]

$$
\zeta, \eta \in S, \quad d(\zeta, \eta)=|1-\bar{\zeta} \cdot \eta|
$$

$d$ est invariante sous l'action de $S U(n)$. Cette pseudo-distance vérifie

$$
\exists K>0 \text { t.q. } \forall \zeta, z, z^{\prime} \in S \text { on a: } d\left(\zeta, z^{\prime}\right) \leqslant K\left[d(\zeta, z)+d\left(z, z^{\prime}\right)\right] .
$$

Posons, pour $\zeta \in S$ et $h>0, R(\zeta, h)=\{\eta \in S / d(\zeta, \eta)<h\}$. On a alors

$$
\forall \zeta \in S, \forall h>0, \quad \sigma_{n}\{R(\zeta, h)\} \simeq h^{n}
$$

où $\sigma_{n}$ est la mesure de Lebesgue sur $S=\partial \mathbf{B}_{n}$. Il existe $K^{\prime}>0$ t.q. $\forall h>0, \exists N_{h} \in \mathbf{N}$ et un réseau $\mathscr{F}_{h}=\left\{\zeta_{k}, 1 \leqslant k \leqslant N_{h}\right\}$ de points de $S$ vérifiant [1, Chapter IV]:

$$
\left\{\begin{array}{l}
R\left(\zeta_{k}, h\right) \cap R\left(\zeta_{l}, h\right)=\varnothing, \quad k \neq l, \\
\cup_{k}\left(\zeta_{k}, K^{\prime} h\right)=S .
\end{array}\right.
$$

Soit $\zeta_{k} \in \widetilde{F}_{h} \cap R(\zeta, s)=E$, où $\zeta \in S$, et $s>h$ et soit $z \in R\left(\zeta_{k}, h\right)$, par (2.2) on a

$$
d(\zeta, z) \leqslant K\left[d\left(\zeta, \zeta_{k}\right)+d\left(\zeta_{k}, z\right)\right] \leqslant K[h+s] \leqslant 2 K s,
$$

d'où: $R\left(\zeta_{k}, h\right) \subset R(\zeta, 2 K s)$. Par (2.3) et (2.4), on en déduit

$$
\sum \sigma_{n}\left(R\left(\zeta_{k}, h\right)\right) \leqslant \sigma_{n}\{R(\zeta, 2 K s)\}
$$

et donc par (2.3)

$$
\operatorname{Card}\left\{\mathscr{F}_{h} \cap R(\zeta, s)\right\} \lesssim s^{n} / h^{n}
$$

Supposons maintenant $s \geqslant 2 K K^{\prime} h$. La pseudo-boule $R\left(\zeta, \frac{s}{2 K}\right)$ ne peut être recouverte par des pseudo-boules de rayons $K^{\prime} h$ que si leur centre est dans $R(\zeta, s)$; en effet, soit $z \in R\left(\zeta, \frac{s}{2 K}\right), z^{\prime} \notin R(\zeta, s)$ on a par (2.2)

$$
d\left(\zeta, z^{\prime}\right) \leqslant K\left[d(\zeta, z)+d\left(z, z^{\prime}\right)\right]
$$

d'où

$$
d\left(z, z^{\prime}\right) \geqslant \frac{1}{K} d\left(\zeta, z^{\prime}\right)-d(\zeta, z) \geqslant \frac{s}{K}-\frac{s}{2 K}=\frac{s}{2 K} \geqslant K^{\prime} h .
$$

D'autre part les pseudo-boules de $\mathscr{F}_{h}$ recouvrent $S$ donc aussi $R\left(\zeta, \frac{s}{2 K}\right)$ d'où; d'après ce qui précède:

$$
\bigcup_{\zeta_{k} \in E} R\left(\zeta_{k}, K^{\prime} h\right) \supset R\left(\zeta, \frac{1}{2 K} s\right) \quad \operatorname{par}(2.4)
$$

et donc:

$$
\operatorname{Card}\left\{\widetilde{F}_{h} \cap R(\zeta, s)\right\}>\frac{s^{\prime \prime}}{h^{\prime \prime}} \quad \text { pour } s \geqslant 2 K K^{\prime} h .
$$

On en déduit donc:

$$
\forall h>0, \forall s \geqslant 2 K K^{\prime} h, \forall \zeta \in S, \quad \operatorname{Card}\left\{\mathscr{F}_{h} \cap R(\zeta, s)\right\} \simeq\left(\frac{s}{h}\right)^{n}
$$

où $\operatorname{Card}\{E\}$ désigne le nombre d'éléments de $E$. 
Construction. Soit $a$ t.q. $0<a<1$ et considérons les sphères $S_{k}$ suivantes:

$$
S_{k}=\left\{z \in \mathbf{C}^{n},|z|=1-a^{k}\right\}, \quad k=1,2, \ldots
$$

Pour $\delta>0$, sur chaque $S_{k}$ plaçons un réseau $\widetilde{F}_{h}$ avec

$$
h=\delta a^{k} ;
$$

on notera $\widetilde{F}_{k}$ ce réseau sur $S_{k}$ et $\zeta_{k, l}$ le point générique de $\widetilde{F}_{k}$.

On note encore

$$
X_{k, l}=\left\{z \in \mathbf{B}_{n}, \bar{\zeta}_{k, l} . z=\left|\zeta_{k, l}\right|^{2}\right\} .
$$

$X_{k, l}$ est une boule dans $\mathbf{C}^{n}$ de dimension réelle $2 n-2$. On pose enfin

$$
X=\bigcup_{k, l} X_{k, l}
$$

On va montrer que $X$ vérifie les conclusions de la proposition. (A) $\exists p>0$ t.q. $X$ est le zéro d'une fonction de $A^{p}\left(\mathbf{B}_{n}\right)$.

Pour cela on plonge la situation précédente dans la boule de $\mathbf{C}^{n+1}$ en ajoutant une variable supplémentaire $w$ :

$$
\tilde{X}_{k, l}=\left\{(z, w) \in \mathbf{B}_{n+1} / z \in X_{k, l}\right\} ; \quad \tilde{X}=\bigcup_{k, l} \tilde{X}_{k, l} \cdot
$$

On a alors

Lemme. La mesure $\left(1-|z|^{2}-|w|^{2}\right)[\tilde{X}]$ est de Carleson dans $\mathbf{B}_{n+1}$.

Preuve. On note pour $h>0, \zeta \in \partial \mathbf{B}_{n+1}$

$$
Q(\zeta, h)=\left\{\eta \in \mathbf{B}_{n+1} / 1-h \leqslant|\eta|<1 \text { et } \frac{\eta}{|\eta|} \in R(\zeta, h)\right\}
$$

et il nous faut montrer que

$$
\int_{Q(\zeta . h)}\left(1-|z|^{2}-|w|^{2}\right)[\tilde{X}] \lesssim h^{n+1}, \quad \forall \zeta \in \partial \mathbf{B}_{n+1}, \forall h>0 .
$$

Compte tenu de la forme de $\tilde{X}$, on voit aisément qu'il suffit de vérifier (2.10) pour les points $\zeta$ de $\partial \mathbf{B}_{n+1}$ tels que leur $n+1$ ième coordonnée est nulle; on a alors

$$
I=\int_{Q(\zeta, h)}\left(1-|z|^{2}-|w|^{2}\right)[\tilde{X}]=\sum_{k, l} \int_{Q(\zeta, h)}\left(1-|z|^{2}-|w|^{2}\right)\left[\tilde{X}_{k, I}\right]
$$

Pour une raison d'homogénéité de la pseudo-distance $d$, il existe une constante $K$ telle que

$$
\tilde{X}_{k, l} \cap Q(\zeta, h) \neq \varnothing \Rightarrow \zeta_{k, l} \in Q(\zeta, K h) .
$$

De (2.11) et (2.12) on tire donc

$$
\begin{aligned}
I & \leqslant \sum_{\zeta_{k, l} \in Q(\zeta, K h)} \int_{Q(\zeta, h)}\left(1-|z|^{2}-|w|^{2}\right)\left[\tilde{X}_{k, l}\right] \\
& \leqslant \sum_{\zeta_{k, l} \in Q(\zeta, K h)}\left(1-\left|\zeta_{k, l}\right|^{2}\right) \operatorname{vol}\left(\tilde{X}_{k, l}\right) .
\end{aligned}
$$


Mais $\tilde{X}_{k, l}$ est une boule dans $\mathbf{B}_{n+1}$ de dimension $2 n$ et de rayon $\sqrt{1-\left|\zeta_{k, l}\right|^{2}}$ d'où

$$
\operatorname{vol}\left(\tilde{X}_{k, l}\right) \simeq\left(1-\left|\zeta_{k, l}\right|^{2}\right)^{n} \simeq a^{n k}
$$

D'autre part $Q(\zeta, K h)$ induit sur la sous-boule $\{w=0\}$ une pseudo-boule dont la projection sur $\partial \mathbf{B}_{n}$ est $R(\zeta, K h)$ car $\zeta \in \mathbf{B}_{n+1} \cap\{w=0\}$; grâce à (2.5) on a donc

$$
\forall k \in \mathbf{N}, \quad \operatorname{Card}\left\{\mathscr{F}_{k} \cap Q(\zeta, K h)\right\} \lesssim \delta^{-n} h^{n} a^{-n k}
$$

on a donc, en portant dans (2.13)

$$
\left\{\begin{array}{l}
I \leqslant \sum_{\zeta,, I \in Q(\zeta, K h)} a^{k} \cdot a^{n k} \lesssim \sum_{k, a^{k} \leqslant K h} a^{(n+1) k} \sum_{l, \zeta_{k, l} \in Q(\zeta, K h)} 1, \\
I \lesssim \sum_{k, a^{k} \leqslant K h} \delta^{-n} h^{n} a^{(n+1) k} a^{-n k} \lesssim \delta^{-n} h^{n+1} .
\end{array}\right.
$$

$\operatorname{Car} \zeta_{k, l} \in Q(\zeta, K h) \Rightarrow\left|\zeta_{k, l}\right| \geqslant 1-K h \Rightarrow a^{k} \leqslant K h$, d'où le lemme.

Pour achever la preuve du $A$ on utilise alors un théorème de N. Varopoulos [7] qui nous affirme que

$$
\exists p>0 \text { et } f \in H^{p}\left(\mathbf{B}_{n+1}\right) \text { t.q. } \tilde{X}=f^{-1}(0) .
$$

Posons alors $g(z)=f(z, 0), z \in \mathbf{B}_{n+1}$. On a bien

(1) $X=g^{-1}(0)$,

(2) $g \in A^{p}\left(\mathbf{B}_{n}\right)$ grâce au lemme de subordination.

(B) X n'est pas un diviseur de la classe de Nevanlinna. Dans [12] l'auteur montre que la condition

$$
\int_{B}\left(1-|z|^{2}\right)[X]<+\infty
$$

est nécessaire pour que $X$ soit un diviseur de la classe de Nevanlinna, dans [6 et 11] les auteurs montrent, indépendamment, que (2.18) est aussi suffisant, résultat que nous n'utiliseront pas ici. Il nous suffit donc de montrer ici que (2.18) n'est pas satisfait i.e. $\left(1-|z|^{2}\right)[X]$ n'est pas une mesure finie dans $\mathbf{B}$, pour, utilisant [12], obtenir que $X$ n'est pas un diviseur de la classe de Nevanlinna.

On a vu que (1.4)

$$
\int_{\mathbf{B}_{n}}\left(1-|z|^{2}\right)[X]=\int_{\mathbf{B}_{n+1}}[\tilde{X}]=\sum_{k, l} \operatorname{vol}\left(\tilde{X}_{k, 1}\right)=\tilde{T}
$$

mais grâce à (2.4) on a

$$
\mathcal{T}=\sum_{k} a^{n k} \sum_{l} 1 \simeq \sum_{k} a^{n k} a^{-n k}=+\infty
$$

la dernière équivalence grâce à (2.5) en $y$ faisant $s=1$ et $h=\delta a^{k}$.

Pour conclure, on peut poser deux questions: (a) Parmi tous les ensembles analytiques contenant les points de $\bigcup_{k=1}^{k_{0}}, \widetilde{F}_{k}$, l'ensemble $\tilde{X}_{k_{0}}=\bigcup_{k=1.1}^{k_{01}} \tilde{X}_{k .1}$ est-il celui ayant le plus petit volume?

(b) Est-il possible de trouver un diviseur de la classe de Nevanlinna de $\mathbf{B}_{n}$ contenant les points de $\bigcup_{k=1}^{\infty} \widetilde{F}_{k}$ ?

Le rapport avec le problème traité dans ce travail est le suivant. 
Dans [1] on montre que si l'on prend $a>0$ assez petit et $\delta>0$ assez grand alors les points de $\bigcup_{k=1}^{\infty} \mathscr{F}_{k}$ forment une suite d'interpolation pour $A^{2}\left(\mathbf{B}_{n}\right)$ donc aussi que ces points sont contenus dans le zéro d'une fonction de $A^{2}\left(\mathbf{B}_{n}\right)$; si la réponse est non au (b) (ce dont je suis convaincu) alors on a ainsi une autre preuve de la proposition donc du théorème.

\section{Annexe.}

LEMME DE SUbORdinATION. Soit $p>0$ et $f\left(z_{1}, \ldots, z_{n}, z_{n+1}\right)$ une fonction holomorphe dans $H^{p}\left(\mathbf{B}_{n+1}\right)$ alors $g\left(z_{1}, \ldots, z_{n}\right)=f\left(z_{1}, \ldots, z_{n}, 0\right)$ est dans $A^{p}\left(\mathbf{B}_{n}\right)$. Réciproquement si $g\left(z_{1}, \ldots, z_{n}\right)$ est dans $A^{p}\left(\mathbf{B}_{n}\right)$ alors $f\left(z_{1}, \ldots, z_{n+1}\right)=g\left(z_{1}, \ldots, z_{n}\right)$ est dans $H^{p}\left(\mathbf{B}_{n+1}\right)$.

Preuve. On vérifie aisément que le mesure d'intégration $\mu$ sur $\left\{z_{n+1}=0\right\} \cap \mathbf{B}_{n+1}$ est de Carleson dans $B_{n+1}$ et on applique le théorème de Carleson-Hörmander [10]. Pour montrer la réciproque il suffit de décomposer la mesure de surface et d'appliquer Fubini.

Ce lemme lie aussi les noyaux de Bergman de $\mathbf{B}_{n}$ et de Szegö de $\mathbf{B}_{n+1}$ [9] mais nous n'en avons pas besoin ici.

\section{BibliographiE}

1. E. Amar, Suites d'interpolation pour les classes de Bergman de la boule et du polydisque de $C^{\prime \prime}$. Canad. J. Math. 30 (1978), 711-737.

2. B. Berndtsson, Integral formulas and zeros of bounded holomorphic functions in the unit ball, preprint.

3. R. Coifman and G. Weiss, Analyse harmonique non-commutative sur certains espaces homogènes, Lecture Notes in Math., vol. 242, Springer-Verlag, Berlin and New York. 1971.

4. P. Lelong, Fonctionnelles analytiques et fonctions entières. Sém. Math. Sup. (Été 1967), Presses Univ. Montréal, Montréal.

5. W. Rudin, Function theory in the unit ball of $\mathbf{C}^{\prime \prime}$. Die Grundlehren der Math. Wissenschaften, vol. 241, Springer-Verlag, New York, 1980.

6. H. Skoda, Valeurs au bord pour l'opérateur d" et zéros des fonctions de la classe de Nevanlinna, Bull. Soc. Math. France 104 (1976), 225-299.

7. N. Varopoulos, Zeros of $H^{p}$ functions in several complex variables, Publ. Anal. Harm. Orsay 334 (1978).

8. G. H. Hardy and J. E. Littlewood, Some properties of conjugate functions, J. Funct. Math. 167 (1932), 405-423.

9. D. Amar et al., Sur les suites d'interpolation en plusieurs variables, Pacific J. Math. 75 (1978), 15-21.

10. L. Hörmander, $L^{p}$ estimates for ( pluri-) subharmonic functions, Math. Scand. 20 (1967), 65-78.

11. G. M. Henkin, Solutions with estimates of the H. Lewy and Lelong-Poincaré equations, Dokl. Akad. Nauk SSSR 210 (1975), 771-774.

12. Pak Song Chee, The Blaschke condition for bounded holomorphic function, Trans. Amer. Math. Soc. 148 (1970), 248-263.

Departement de Mathematiques, Universite de Bordeaux I, 351, Cours de la Liberation, 33405 TALENCE, France 